\title{
La station-service : étude spatiale du réseau de distribution de carburant à Strasbourg
}

The service station: a spatial study of petrol distribution in Strasbourg

Die Tankstelle: Raumstudie eines Verteilernetzes für Treibstoff in Strassburg

\section{Claire Hiegel}

\section{(2) OpenEdition}

Journals

Édition électronique

URL : http://journals.openedition.org/rge/4444

ISSN : $2108-6478$

Éditeur

Association des géographes de l'Est

Édition imprimée

Date de publication : 1 avril 1999

ISSN : 0035-3213

Référence électronique

Claire Hiegel, « La station-service : étude spatiale du réseau de distribution de carburant à

Strasbourg ", Revue Géographique de l'Est [En ligne], vol. 39 / 2-3 | 1999, mis en ligne le 05 septembre 2013, consulté le 07 septembre 2020. URL : http://journals.openedition.org/rge/4444

Ce document a été généré automatiquement le 7 septembre 2020

Tous droits réservés 


\section{La station-service : étude spatiale du réseau de distribution de carburant à Strasbourg}

The service station : a spatial study of petrol distribution in Strasbourg

Die Tankstelle : Raumstudie eines Verteilernetzes für Treibstoff in Strassburg

Claire Hiegel

1 Nos grands-parents, au début de ce siècle, ont vu apparaître sur les trottoirs, devant les petits commerces, des fûts de carburant dotés d'un système de pompage. Il s'agit là des premières ébauches de stations-service. Aux États-Unis, les réseaux de distribution de carburant voient le jour dans les années 1900-1910, tandis qu'en France et dans le reste de l'Europe, il faut attendre 1920. À ses débuts, la distribution est effectuée dans des locaux non spécifiques, d'un côté, par des commerçants qui détiennent, entre autres, la vente de pétrole lampant (épiciers, cafetiers, quincailliers) et de l'autre, par des professionnels de l'automobile comme les carrossiers. Avec la démocratisation de l'automobile, les postes de distribution prennent place dans le paysage et plus précisément sur le bord des routes. Ils vont cependant subir de multiples modifications avant de devenir les stations-service qui nous sont si familières (G. Dupuy, 1995). Pour F. Nordemann (1981), les premiers postes à essence sont « des dépôts installés sur le passage des voitures » et ne sont rien d'autre que des «solutions simples au problème de la distribution ». Dès les années 1930-1935, les compagnies pétrolières désirent distinguer les postes de carburant des hangars et ateliers réservés jusque là à l'automobile. C'est alors qu'un type de bâtiment spécialisé dans la vente de l'essence fait son apparition : la station-service.

2 L'implantation de telles installations suscita alors une vive hostilité auprès d'une partie de la population à cause du danger qu'elles représentaient (incendie, explosion, pollution, nuisance olfactive), et d'autre part, en raison du manque de réglementation et donc de normes de sécurité. Durant son évolution, la station-service a bénéficié de nombreux progrès techniques et surtout de la mise en place d'une réglementation stricte $^{1}$. Cependant, cette installation est encore actuellement à l'origine de nombreux 
accidents. D'après la base de données ARIA ${ }^{2}$, près d'une centaine d'accidents se sont produits dans les stations-service depuis 1988, et selon l'inventaire des accidents industriels de 1990, ces accidents, associés à ceux survenus dans les garages, représentent 4,6\% des accidents industriels, ce qui n'est pas négligeable.

Le risque est certes "mineur ", mais constamment présent du fait de l'imbrication des stations-service dans le bâti urbain. Cette notion de proximité est essentielle dans l'étude des risques technologiques car elle peut être à l'origine de nombreux accidents, mais elle peut aussi expliquer la potentialité d'occurrence et l'impact potentiel de celuici. Pour étudier et analyser les risques, il est essentiel d'une part, de les identifier et de les localiser et d'autre part, de prendre connaissance de l'environnement dans lequel ils s'intègrent. Cet article fait suite à un travail universitaire (C. Hiegel, 1995), sur l'analyse des risques technologiques "mineurs" liés aux stations-service, qui a abouti à une identification des risques, à une analyse de la localisation des installations et à la mise en place d'une typologie des stations et des risques présentés par ces dernières. Il s'agit, outre la présentation générale de l'équipement en pompes à essence de la Communauté Urbaine de Strasbourg (CUS), d'appréhender leur répartition et d'en proposer une typologie. L'aspect «identification» des risques n'est pas abordé. L'article présente surtout une première approche méthodologique du risque technologique mineur au travers d'une analyse spatiale et d'une typologie.

\section{Un premier bilan}

4 La Communauté Urbaine de Strasbourg regroupe 27 communes hétérogènes qui comptabilisaient en 1990423712 habitants sur une superficie de $305,81 \mathrm{~km}^{2}$. Ces communes présentent, soit un "caractère villageois", soit un "caractère urbain prononcé» (R. Kleinschmager, 1997). Les unes sont vastes comme Strasbourg, Hoenheim ou encore La Wantzenau, tandis que d'autres sont nettement plus petites comme Mittelhausbergen ou encore Niederhausbergen. Elles se différencient également par leur densité de population : quelques unes dépassent 3000 habitants au $\mathrm{km}^{2}$ (Fig. 1a). 
Figure 1a : Communauté Urbaine de Strasbourg

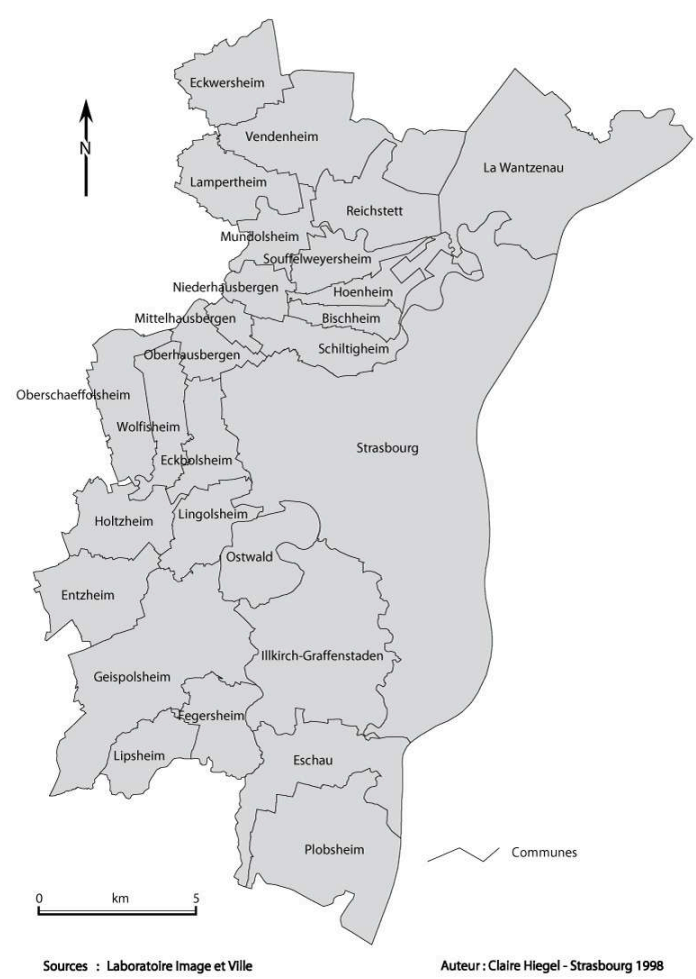

5 L'hétérogénéité de ces communes fait que la CUS possède aussi un réseau routier diversifié. En l'occurrence, elle dispose d'autoroutes (Paris-Strasbourg et StrasbourgMulhouse) fort bien relayées par des nationales. Plusieurs communes sont traversées par ces voies de communication qui enregistrent un trafic important sans compter les rues, avenues et boulevards qui drainent des flux différenciés de véhicules (Fig. 1b). 
Figure 1b : Communauté Urbaine de Strasbourg : infrastructures

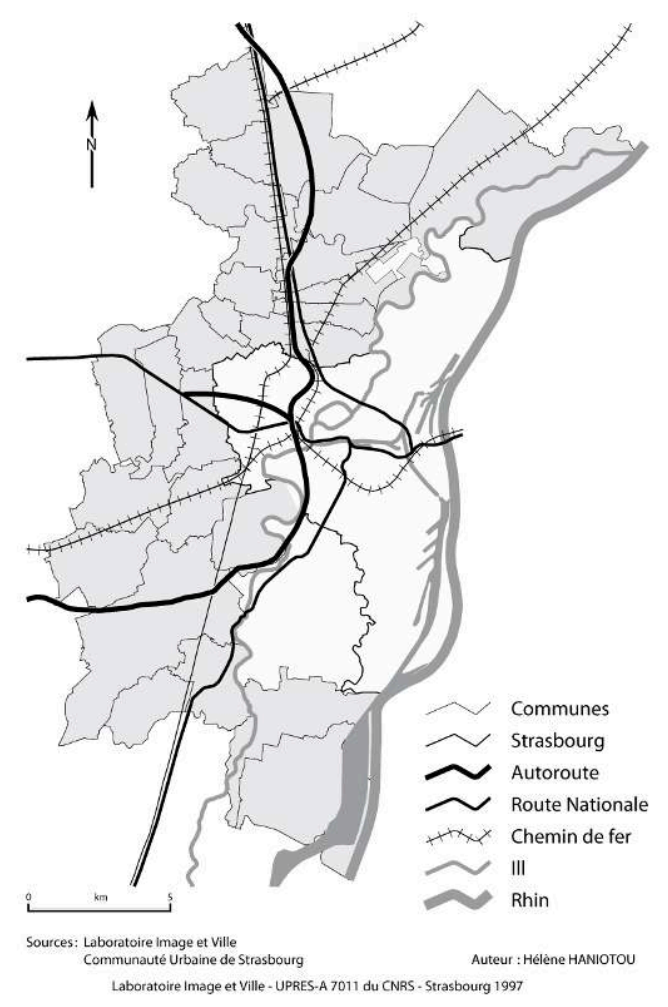

6 De par le caractère hétérogène des communes et la diversité du réseau routier, la Communauté Urbaine de Strasbourg constitue une zone intéressante pour l'analyse d'un réseau de distribution de carburant.

\section{A. À la recherche des stations-service}

7 Recenser les stations-service n'est pas chose aisée en raison du manque d'informations disponibles qui s'explique par l'existence de divers types de distributeurs : les stationsservice officielles qui appartiennent aux grandes compagnies pétrolières, les clients organiques regroupant des particuliers (essentiellement des professionnels de l'automobile comme les garagistes et les concessionnaires), qui ont passé un contrat avec les pétroliers afin de pouvoir distribuer du carburant, et enfin, les stationsservice de supermarché.

La principale difficulté réside dans le fait que les clients organiques et les stationsservice de supermarché ne sont pas répertoriés comme distributeurs de carburant, mais sous la dénomination de leur activité principale. C'est pourquoi la Chambre de Commerce et d'Industrie de Strasbourg ne possède pas de liste répertoriant les trois types de distributeurs. Cependant, les stations-service stockent et distribuent des liquides inflammables, ce qui fait d'elles des «Installations Classées » pour la protection de l'environnement recensées par la DRIRE $^{3}$; mais n'atteignant pas toutes le seuil de stockage considéré comme potentiellement dangereux, elles n'y sont pas toutes enregistrées. Cette difficulté a nécessité l'élaboration d'une base de données constituée à l'aide des pages jaunes de l'annuaire de France Télécom et des divers listings (garagistes, concessionnaires, supermarchés, stations officielles) obtenues à la Chambre de Commerce. Un travail de terrain s'est avéré indispensable afin de vérifier quels sont, 
parmi les clients organiques et les supermarchés, ceux qui distribuent réellement du carburant.

9 Ce recensement a abouti à une base de données localisées qui comptabilise pour la Communauté Urbaine de Strasbourg 73 stations-service en 1995.

\section{B. Le réseau de distribution : une nouvelle donne}

Les stations-service étaient bien plus nombreuses dans la CUS avant 1995, mais pour des raisons de sécurité, d'esthétique, de nuisance pour le voisinage et sans compter la concurrence accrue par la proximité de Kehl (Allemagne) qui propose des prix attractifs, plus de la moitié d'entre elles ont disparu. Les plus touchées par la vague de fermeture ont été les stations-service officielles et bon nombre d'entre elles ont fermé à Strasbourg, essentiellement dans les quartiers centraux (J. Blaesius, 1992).

11 Alors que certaines ont disparu, d'autres ont fait leur apparition : les stations-service officielles de «l'an 2000 » (comme se plaît à le dire un pompiste strasbourgeois). Ces dernières ont entièrement été restructurées et modernisées pour attirer les automobilistes. Les pétroliers misent sur un nouveau design, davantage d'espace et des services variés : lavage de voiture, supérette, paiement par carte, entretien automobile, buffet. À côté de ces stations de "l'avenir», coexistent d'autres postes à essence importants, tels ceux localisés sur les bords des autoroutes, qui, en plus des divers services proposés, mettent à la disposition des clients des aires de repos et occupent ainsi une place d'envergure dans le paysage.

12 Par ailleurs, depuis une vingtaine d'années, on voit apparaître sur le marché du carburant, un «monstre » de la distribution: le supermarché. Sur le territoire de la CUS, les grandes surfaces représentent souvent les plus grandes stations d'alimentation en essence. C'est le cas notamment de Cora dans la zone commerciale de Mundolsheim qui totalise 13 postes de distribution, tandis qu'une station dite de «l'an 2000 » en compte généralement 4 ou 5 . Ces stations de supermarché représentent aujourd'hui environ un quart des postes d'alimentation en essence au détriment des stationsservice officielles.

13 À côté de ces « monstres » de la distribution, les clients organiques sont nettement plus modestes. Ils sont surtout présents dans les petites communes où ils constituent souvent les seuls postes d'alimentation en essence. Tout comme les stations-service officielles, ils doivent faire face à la concurrence accrue des stations de supermarché. Bon nombre d'entre eux ont ainsi été contraints à la fermeture.

14 L'évolution du réseau de distribution de carburant de la Communauté Urbaine de Strasbourg ne lui est pas propre : elle suit parfaitement la tendance française dont le fait majeur est la fermeture de nombreuses pompes et principalement celles des stations-service officielles. Après avoir connu un essor formidable à partir de la fin des années 1920, ces dernières voient leur parc réduit de moitié, passant en France de 47500 points de vente en 1976 à 31500 en 1988 (J.-M. Calvat, 1988). La concurrence, qui a toujours été rude entre les compagnies pétrolières, s'est accrue avec l'ouverture des postes à essence dans les grandes surfaces, dont les effectifs ont doublé en sept ans ( 2650 postes en 1987 contre seulement 1290 en 1980). Ces espaces commerciaux représentent aujourd'hui un tiers des postes de distribution en France. Face à une telle compétition, les compagnies pétrolières se voient dans l'obligation de remanier leur réseau de distribution, en commençant par réduire le nombre de leurs stations-service. 
Cette évolution est identique sur le territoire de la CUS où la suppression des pompes laisse certaines communes totalement démunies en carburant, obligeant ainsi leurs habitants à parcourir plusieurs kilomètres pour se ravitailler en essence.

\section{Une distribution inégale}

Plusieurs constats peuvent être énoncés avant même de procéder à une analyse spatiale de la répartition des postes de carburant sur le territoire de la CUS.

\section{Premier constat : la prépondérance des stations-service officielles}

Parmi les 73 stations-service, 40 sont officielles, 17 appartiennent à des supermarchés et 16 sont tenues par des clients organiques. Elles représentent respectivement $55 \%$, $23 \%$ et $22 \%$ des postes de distribution de carburant sur le territoire de la CUS. Les stations-service officielles, malgré les nombreuses fermetures, restent les postes les plus nombreux.

Deuxième constat : la relation entre le nombre de stations-service et la population des communes (tableau 1)

18 Il existe manifestement un lien entre le nombre d'habitants par communes et le nombre de postes de distribution de carburant.

Tableau 1 : Population communale et stations-service

\begin{tabular}{|l|c|}
\hline Nombre d'habitants & Nombre de stations \\
\hline Moins de 3000 & 0 \\
\hline De 3000 à 4999 & 1 \\
\hline De 5000 à 9999 & 2 \\
\hline De 10000 à 19999 & 3 ou 4 \\
\hline De 20000 à 30000 & 5 \\
\hline Plus de 30 000 & 30 \\
\hline
\end{tabular}

19 Le tableau 1 montre qu'il n'y a pas de station-service dans les communes de moins de 3000 habitants. Ce chiffre semble être un seuil. En effet, à l'exception d'Eckbolsheim, toutes les municipalités de 3000 habitants et plus sont dotées de postes à essence tandis que celles de moins de 3000 habitants en sont dépourvues. Avec 255937 habitants, Strasbourg est la commune la mieux équipée puisqu'on y recense une trentaine de stations. En fait, le nombre de stations-service va croissant en fonction de l'augmentation du nombre d'habitants dans les communes.

\section{Troisième constat : l'inégale répartition des stations-service}

Sur les 27 communes de la CUS, 8 ne sont pas équipées en postes à essence par opposition à Illkirch-Graffenstaden et Schiltigheim qui en totalisent 5 et enfin Strasbourg qui en recense 30 . Cette dernière concentre ainsi $41 \%$ des stations-service de la CUS, dont $55 \%$ sont des postes officiels, $22 \%$ des pompes de supermarché et $23 \%$ des clients organiques. Cette inégale répartition entre les communes s'explique en partie par la relation qui existe entre le nombre de postes de carburant et le nombre 
d'habitants. Mais une analyse spatiale du réseau de distribution permettra de mieux déterminer les localisations des stations-service et d'examiner leur répartition dans l'espace de la Communauté Urbaine de Strasbourg.

Ce bilan présente un état des lieux rapide du réseau de distribution de carburant de la CUS. A présent, et toujours dans l'objectif de l'étude des risques liés aux stationsservice, il s'agit de localiser ces installations «dangereuses » et d'analyser leurs positions respectives

\section{La répartition des stations-service dans la CUS}

Avec le développement de l'automobile, la création des grands axes routiers, ces établissements à risque se sont très largement intégrés dans le milieu urbain, et certaines méthodes permettent une appréhension quantifiée et comparable de leur distribution spatiale.

\section{A. Les techniques d'analyse (d'après C. Cauvin et S. Rimbert, 1976)}

L'analyse spatiale de la répartition des stations-service a été effectuée à l'aide de deux familles de méthodes. La première famille utilise directement les semis de points et prend en compte des méthodes d'analyse descriptive, tels que le centre moyen, la distance de Bachi et l'ellipse standard. Rappelons que le centre moyen dégage la tendance centrale du semis par un indice unique ; il est complété par la distance de Bachi qui présente la dispersion des points par rapport à la tendance centrale et donc module le résultat du centre moyen. Enfin, l'ellipse standard permet de mesurer et de résumer la dispersion d'un semis de points en apportant des précisions sur les directions privilégiées de la dispersion.

La seconde famille de méthodes appréhende les localisations à partir de partitions de l'espace introduisant ainsi la notion de zone de densité associée à un point et permet de plus, d'utiliser un facteur visuel discriminant. Les polygones de Thiessen consistent à construire une partition de l'espace à partir d'un semis de points en ne tenant compte que de la proximité, délimitant ainsi la « zone d'appartenance de proximité » de chacun des points

Ces techniques fournissent des indices descriptifs illustrés par des cartes qui facilitent la description et les comparaisons éventuelles des différents semis de points représentant la distribution des stations-service dans la Communauté Urbaine de Strasbourg.

\section{B. Les stations-service : une couverture incomplète de la CUS}

Le semis de points indique une concentration des stations-service dans la partie centrale de la CUS, le long de deux axes nord-sud de part et d'autre du centre strasbourgeois. Ceci s'explique d'une part par la structure du réseau routier et d'autre part par la morphologie urbaine du centre-ville. Par contre à la périphérie, elles sont plus rares (fig. 2). 
Figure 2 : Communauté Urbaine de Strasbourg : les stations-service

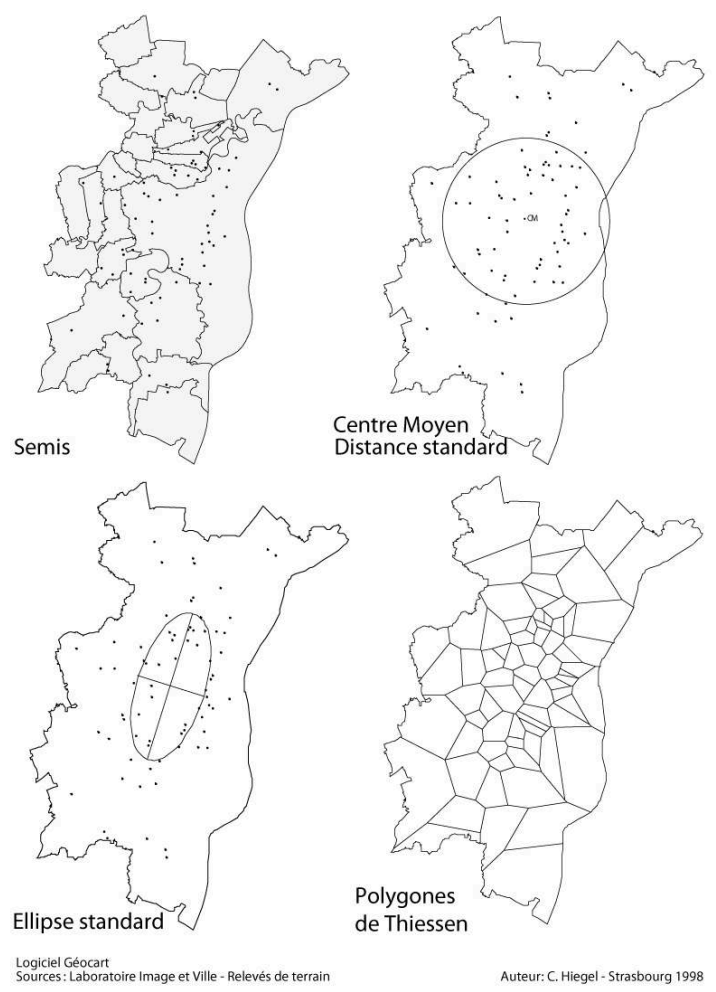

\section{Une position centrale par rapport à la périphérie}

27 Le centre moyen associé à la distance de Bachi indique la centralité du semis et donc la partie de la CUS la mieux équipée en stations-service. L'indice du CM se situe au centre de la Communauté Urbaine, avec une forte concentration de postes à essence dans la commune même de Strasbourg.

Ce résultat doit être nuancé par l'analyse des points autour de ce centre à l'aide de la distance de Bachi et de l'ellipse standard.

\section{Une dispersion assez grande}

La distance de Bachi donne une valeur globale de la dispersion de $5590 \mathrm{~m}$. Cette valeur traduit une grande dispersion des stations-service qui sont donc très largement éparpillées sur l'ensemble du territoire. Une telle valeur doit cependant être complétée par des indices prenant en compte la direction de la dispersion. On utilise pour cela la technique de l'ellipse standard.

Le tracé de l'ellipse prend une forme allongée avec un axe d'étirement principal orienté nord-sud. Ceci indique la direction privilégiée du semis avec plus précisément une orientation générale nord-nord-est, sud-sud-ouest. Plusieurs facteurs peuvent expliquer cette orientation :

- la présence à l'est de la frontière franco-allemande qui biaise la dispersion ;

- la localisation des communes les plus urbaines qui se trouvent au nord et au sud de Strasbourg (Schiltigheim, Hoenheim et Bischheim au nord, et Illkirch-Graffenstaden au sud) ; 
-à l'ouest, enfin, les communes qui ont un caractère plus rural et sont donc moins densément peuplées.

31 Par ailleurs, la surface de l'ellipse, de $37 \mathrm{~km} 2$, recouvre une partie importante des principales communes de la CUS, confirmant ainsi l'importance de la dispersion.

\section{Le bon équipement des communes centrales}

La partition spatiale obtenue avec les polygones de Thiessen met en évidence les zones où les stations-service sont les plus concentrées. Les surfaces après tessellation (ou pavage) font nettement ressortir cette concentration qui se situe au centre de la CUS et concerne les principales communes urbaines centrales. En s'éloignant vers la périphérie, les polygones augmentent de taille et montrent que les stations-service y deviennent plus rares.

Cette première analyse exprime une très nette concentration des postes à essence dans les communes principales. La concentration la plus évidente se fait à Strasbourg, puis dans les communes avoisinantes comme Schiltigheim, Bischheim et Hoenheim au nord, Illkirch-Graffenstaden au sud. Par opposition, les communes périphériques sont nettement moins bien équipées, voire totalement dépourvues de station-service. La couverture est de ce fait incomplète et inégale dans la CUS. Les installations à risque sont donc plus nombreuses au sein de communes très densément peuplées ce qui, en cas d'accident, peut aggraver les conséquences.

\section{Une répartition différenciée (fig. 3)}

La majeure partie des stations-service se concentre dans le centre de la CUS. En est-il de même pour chaque type de distributeur? La couverture de la Communauté Urbaine est-elle identique pour les stations officielles, celles des supermarchés ou encore des clients organiques ? (fig. 3).

Figure 3 : Communauté Urbaine de Strasbourg : localisation par types de stations-service

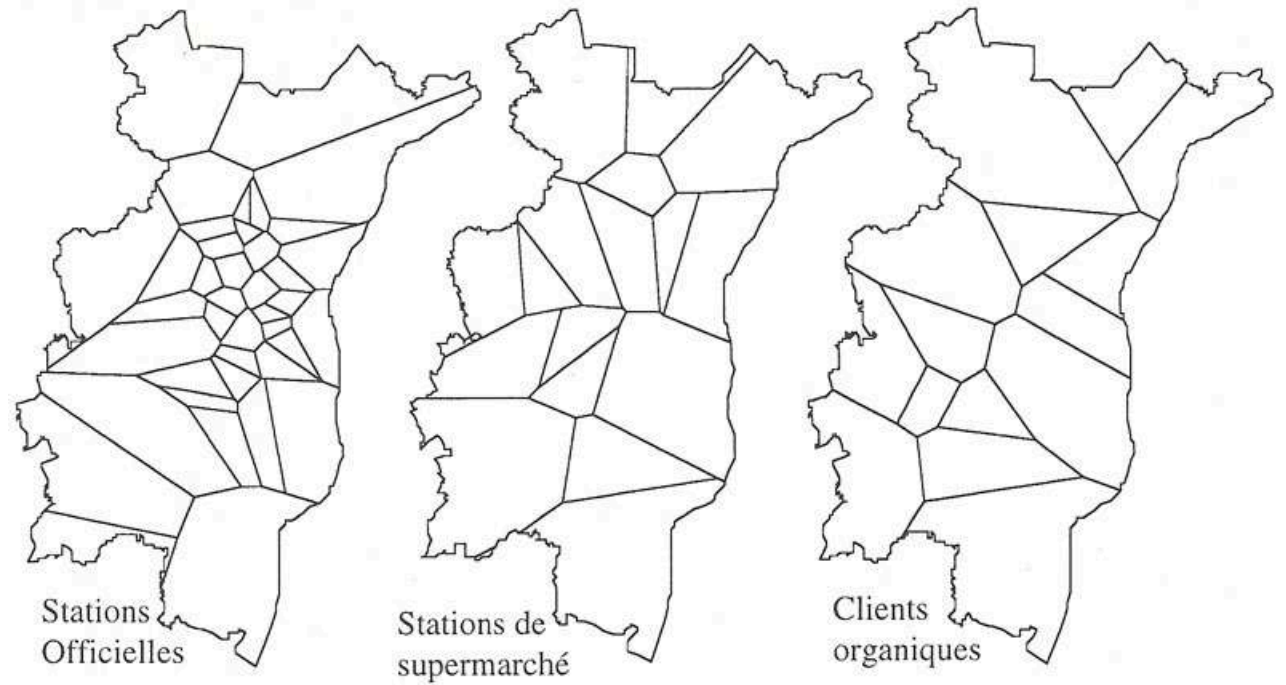

Logiciel Géocart

Sources: Laboratoirc Image et Ville relevés de terrain-C. Hiegel 


\section{Les stations-service officielles} territoire étudié et une direction privilégiée nord-sud. Les partitions confortent la position centrale, avec un fort contraste par rapport à une périphérie sous-équipée, ce qui donne une impression de vide. L'activité principale, voire unique de ce type d'installation est la distribution de carburant. Donc pour être rentable elle doit bénéficier d'un important flux d'automobilistes. De ce fait, elle s'installe le long d'axes majeurs comme les nationales, les autoroutes, ou encore les pénétrantes des centrevilles. Dans cette partie de la CUS fort bien équipée en postes à essence, le réseau de voirie est dense car beaucoup d'axes principaux convergent ou divergent à partir de Strasbourg et traversent alors les communes les plus proches et les plus urbaines.

\section{Les stations de supermarché}

La carte de localisation de ces stations offre un semis très lâche sur pratiquement tout le territoire de la CUS. Cependant, elles sont totalement absentes du centre contrairement au cas précédent. Effectivement, les supermarchés équipés en pompes à essence gravitent autour de Strasbourg, en périphérie, sous la forme d'un demi-cercle avec des extensions vers le nord et vers le sud. Aucune concentration n'est réellement visible, mais on constate toutefois une certaine proximité des stations-service aux abords de la ville de Strasbourg. Il est à noter également que bon nombre de ces stations sont situées aux extrémités nord et sud de la CUS intégrées dans des zones commerciales ou industrielles comme à Vendenheim ou encore à Geispolsheim. L'analyse est ici rendue complexe d'une part en raison du peu de points constituant le semis, et, d'autre part, parce que l'implantation de ces stations-service ne répond sans doute pas à une logique de réseau de distribution de carburant, mais à une logique de grande surface alimentaire.

\section{Les clients organiques}

Il est très difficile de discerner une tendance générale dans ce semis en raison du petit nombre de clients organiques dans la CUS (une quinzaine). Il se distingue cependant par une répartition linéaire qui traverse le territoire du nord-est au sud-ouest, en passant à l'ouest du centre de la CUS, ce qui signifie qu'en plus des communes urbaines, les communes plus petites, à caractère rural, sont dotées, elles aussi, de clients organiques.

Chaque type de station-service se caractérise ainsi par une configuration quelque peu différente. Les stations-service officielles se concentrent au centre de la CUS, les stations de supermarché gravitent autour de ce centre tandis que les clients organiques ne dégagent pas de réelle tendance. Cependant, la dispersion générale des semis est dominée par la direction nord-sud, ce qui correspond à la forme allongée de la CUS, et également à la direction générale de l'emprise urbaine.

L'analyse de la répartition des stations-service met en évidence l'imbrication du risque technologique dans un tissu urbain dense. Cette proximité entre l'installation à risque, le bâti et la population peut s'avérer dangereuse en cas d'accident à " effet de chaîne "

Revue Géographique de l'Est, vol. 39 / 2-3 | 1999 
ou « effet domino ». Cependant, le risque est inégal, car les stations-service diffèrent et le milieu dans lequel elles s'intègrent varie.

\section{Une diversité des risques}

Certaines stations-service sont grandes, d'autres sont plus petites, quelques-unes sont anciennes tandis que d'autres sont modernes. Au bord des autoroutes ou des nationales, en milieu urbain ou en milieu rural, entourées de champs ou cernées par des pavillons..., autant de stations-service et de risques différents qu'il est intéressant d'étudier.

\section{A. Des établissements aux attributs divers}

41 Afin de prendre en compte toute cette diversité, une fiche de relevé a été établie pour chacune des 73 stations-service de la Communauté Urbaine de Strasbourg. Elle comprend des renseignements sur les différentes caractéristiques des stations-service et des informations concernant leur environnement proche. Elle va permettre à l'aide d'un outil statistique - l'analyse des correspondances multiples (ACM) associé à une classification - de proposer une typologie des stations-service dans la CUS. A cet effet, 49 variables ont été retenues :

Une première catégorie de variables concerne les caractéristiques propres à la stationservice :

- le type de station (officiel, supermarché, client organique);

- la marque du carburant (s'il y en a une) ;

- le nombre de pompes ;

- l'accès aux pompes (en libre-service, présence d'un pompiste) ;

- les heures d'ouverture;

- les activités annexes à la vente de carburant (vente de produits alimentaires, restauration rapide, entretien de véhicules, ...).

Une seconde catégorie de critères concerne les caractéristiques de l'environnement de la station-service :

- le type de voirie (rue, nationale, autoroute...) ;

- le type d'habitat environnant (pavillonnaire, collectif...) ;

- les activités aux alentours de la station (industries, commerces, écoles...);

- le type de végétation;

- la densité de population.

Ces différentes variables - toutes n'étant pas présentées ici - et l'utilisation de l'ACM ont permis d'identifier différents types de stations-service.

\section{B. Des groupes à risques inégaux}

La classification associée à l'ACM a permis d'identifier 5 groupes distincts.

- Une première classe sépare les stations-service des grandes surfaces des stations officielles. Les stations de supermarché se caractérisent bien sûr par la présence d'un commerce qui est le supermarché lui-même, et par l'absence de vente de produits d'entretien et d'un atelier de réparation pour véhicules. Par opposition à ce type de 
stations, les secondes possèdent tous les services annexes à la vente de carburant que ne proposent pas les stations de supermarché. Ces deux types de stations se répartissent dans la commune strasbourgeoise pour les stations officielles, et en périphérie de la cité pour les stations de supermarché.

- Une seconde catégorie distingue encore les stations de supermarché, non plus en fonction de leurs services, mais du milieu dans lequel elles se sont implantées. Il s'agit là des pompes situées dans les zones commerciales, n'ayant aucun habitat aux alentours et munies de plus de 6 pompes. Ce sont des stations de grande taille comme celles de Cora (Mundolsheim), Auchan (Strasbourg Hautepierre) et Rond-Point (Geispolsheim). Elles se localisent à la périphérie de la Communauté Urbaine ou encore dans les quartiers limitrophes de la ville. Cette même classe comprend également les stations-service, qu'elles soient officielles ou tenues par des clients organiques, dont le nombre de pompes est compris entre 3 et 6 et qui sont implantées dans des quartiers résidentiels. En ce qui concerne leur localisation, elle est nettement plus diffuse sur le territoire de la CUS.

- Un autre groupe inclut les stations-service implantées le long des routes nationales, qui ne proposent aucun service en dehors de la distribution de carburant et autour desquelles il n'y a pas d'habitat. Elles se trouvent tels des blocs isolés dans la nature, comme par exemple la station de Wolfisheim le long de la RN 4. Les stations entourées de commerces, d'habitats collectifs et qui proposent de nombreux services en annexe, s'opposent à cette catégorie. Elles se situent essentiellement dans les communes à caractère urbain et dans les quartiers densément peuplés comme par exemple dans celui de l'Esplanade à Strasbourg.

- Une quatrième famille met en avant les stations qui proposent un buffet et donc un moyen de restauration rapide. Tous les autres postes à essence s'opposent à ces dernières. Il n'y a en effet que les stations situées le long des autoroutes qui proposent ce type de service. Dans la Communauté Urbaine de Strasbourg deux importantes stations autoroutières sont implantées aux bords de l'A 35 : l'une dans la direction Strasbourg-Mulhouse et l'autre dans le sens Strasbourg-Paris.

50 - Enfin une dernière classe distingue les stations des clients organiques des autres types de stations-service. Les premières disposent d'un faible nombre de pompes, inférieur à 3, mais un pompiste sert les clients; de plus, elles proposent un atelier de réparation de véhicules. Cependant, ces stations ne vendent ni produits alimentaires, ni gaz et ne possèdent pas de centre de lavage pour voiture. Elles se situent surtout dans les communes de la CUS à caractère rural comme par exemple La Wantzenau, Geispolsheim ou encore Plobsheim. À l'opposé des clients organiques, on trouve les deux autres types de stations service qui offrent de nombreux services à leurs clients tels le lavage de voiture et la vente de bouteilles de gaz.

51 Cette typologie va au-delà d'une simple distinction entre les types «officiels » (organique, officielle et de supermarché). Elle permet également de mettre en avant une inégalité des risques en fonction de l'environnement dans lequel s'est implantée l'installation.

52 Une station de supermarché comportant un grand nombre de pompes implique nécessairement des quantités de stockage et un débit importants et représente de ce fait un risque non négligeable. Cependant, ce type de station est souvent localisé dans des zones commerciales où les bâtiments sont éloignés les uns des autres et sans habitat à proximité. L'installation est de ce fait moins dangereuse qu'un client 
organique avec deux pompes, mais intégré dans un immeuble de 4 à 5 étages. Cette typologie demande à être améliorée en y apportant des variables supplémentaires comme, par exemple, les distances, le nombre de ménages. En l'état, elle n'est qu'une introduction à l'analyse des risques.

\section{Conclusion}

La Communauté Urbaine de Strasbourg connaît donc une forte concentration de stations-service en son centre dans les communes densément peuplées à trafic routier important. Par contre les postes à essence se raréfient et disparaissent à mesure que l'on s'éloigne de la cité strasbourgeoise qui est la mieux équipée. Les postes de distribution de carburant les plus représentés sont les stations-service officielles, mais le réseau est en pleine mutation avec l'arrivée sur le marché des stations-service de supermarché et de nombreuses fermetures. Cela doit sans aucun doute se répercuter sur la localisation des stations-service. Il serait intéressant d'effectuer une analyse spatiale diachronique afin d'étudier l'évolution de la répartition spatiale des stationsservice dans la CUS. D'autre part, la typologie proposée met en avant la grande diversité des stations-service dans la CUS en fonction de leurs caractéristiques propres et de leur environnement.

Cette analyse de la répartition spatiale des stations-service et l'élaboration de la typologie ont constitué une première approche d'un type de risque technologique. Il s'agissait en effet d'une étude sur la localisation d'installations susceptibles d'être dangereuses. Les risques liés aux stations-service peuvent être qualifiés de "mineurs ", car leurs impacts sont limités dans le temps et dans l'espace. L'existence de ce type de risque est masquée par l'importance donnée aux risques technologiques «majeurs »importance justifiée par l'ampleur des phénomènes en cas d'accident - bien qu'ils soient eux aussi produits par le dysfonctionnement ou les usages mal maîtrisés des technologies actuelles. En outre, les risques « mineurs », très largement imbriqués dans le tissu urbain, sont banalisés par nos sociétés actuelles. De par cette "proximité » il serait bon d'approfondir l'étude sur l'environnement proche de telles installations afin de prévenir les risques à « effet de chaîne ».

\section{BIBLIOGRAPHIE}

Blaesius J. (1992). - « Stations hors service », Dernières nouvelles d'Alsace, 6 décembre, p. 44.

Calvat J. M. (1988). - « Les stations-service disparaissent », Inventaire communal de 1988, pp. 23-26.

Cauvin C., Rimbert S. (1976). - Les méthodes de la cartographie thématique. Fascicule 1 : Lecture numérique des cartes thématiques, Éd. Université de Fribourg, Suisse, 172 p.

Dupuy G. (1995). - Les Territoires de l'automobile, Paris, Anthropos Economica, 216 p. 
Hiegel C. (1995). - Au risque de la ville... Les stations-service à Strasbourg. Un risque technologique mineur. Mémoire de DEA, UFR Géographie Strasbourg, $126 \mathrm{p}$.

Kleinschmager R. (1997). - Strasbourg. Une ambition européenne, Paris, Anthropos, Collection

«Villes ", 216 p.

Nordemann F. (1981). - Bâtiments de l'automobile, Paris, Seuil, 54 p.

\section{NOTES}

1. La loi du 19 juillet 1976 relative aux installations classées pour la protection de l'environnement.

2. "Analyse, recherche, information sur les accidents et pollutions industrielles ». Service chargé de rassembler et analyser les incidents technologiques en France et à l'étranger, dans le but de mieux connaître et donc prévenir les accidents.

3. Direction Régionale de l'Industrie de la Recherche et de l'Environnement.

\section{RÉSUMÉS}

Le développement de l'automobile a fait apparaître un nouveau type de bâtiment spécialisé dans la vente de carburant : la station-service. Dès le début du siècle, elle prend rapidement place dans le paysage et devient un lieu de la quotidienneté où se côtoient les citoyens. Cependant, une telle installation, aujourd'hui banalisée, représente un risque de par le stockage et la distribution de liquides inflammables. Pour gérer et prévenir les risques technologiques, il est nécessaire de les identifier, de les localiser et de les intégrer au sein de leur environnement. Cet article présente, à travers l'étude du réseau de distribution de carburant dans la Communauté Urbaine de Strasbourg, la répartition et la diversité des stations-service.

Development of the automobile required a new type of building specializing in the sale of fuel : the petrol station. Since the beginning of this century, it rapidly took its place in the urban landscape where it is close to the public. Nevertheless, such an installation, quite common today, represents a technical hazard because of the storage and distribution of inflammable liquids. In order to manage and prevent technological hazards it is necessary to identify them, to locate them as well as to integrate them within their environment. This paper deals, through a thorough study of the petrol distribution network in the Communauté Urbaine de Strasbourg, with the spatial distribution and diversity of the petrol stations.

Die Entwicklung des Kraftfahrzeugs liess einen neuen Typ des spezialisierten Gebäudes für den Verkauf von Treibstoff entstehen : die Tankstelle. Seit der Jahrhundertwende gewinnt sie zunehmend an Raum in der Landschaft und wird zu einem Ort des Alltäglichkeit, wo sich die Bürger begegnen. Indessen stellt eine solche heute banale Einrichtung wegen der Lagerung und Verteilung feuergefährlicher Flüssigkeiten ein Risiko dar. Um die technischen Risiken zu erfassen und zu beherrschen ist es notwendig, sie zu identifizieren, zu lokalisieren und in ihr Umfeld zu integrieren. Dieser Artikel stellt mit der Studie über das Netz der Treibstoffausgabe in der Stadtgemeinde von Strassburg die Verteilung und Verschiedenartigkeit der Tankstellen vor. 
INDEX

Schlüsselwörter : Raumanalyse, Strasbourg, Tankstelle, technologisches Risiko

Keywords : service station, spatial analysis, Strasbourg, technological hazard

Mots-clés : analyse spatiale, risque technologique, station-service, Strasbourg

\section{AUTEUR}

\section{CLAIRE HIEGEL}

Laboratoire «Image et Ville »- UPRES-A 7011 CNRS, Université Louis-Pasteur - 3, rue de l'Argonne 67000 Strasbourg 